\title{
TECHNOLOGY DEVELOPMENT ACTIVITIES FOR THE SPACE ENVIRONMENT AND ITS EFFECTS ON SPACECRAFT
}

\author{
B. Kauffman, D. Hardage*, J. Minor, NASA Marshall Space Flight Center, AL 35812 \\ J. Barth, K. LaBel, NASA Goddard Space Flight Center, MD 20771
}

\begin{abstract}
Reducing size and weight of spacecraft, along with demanding increased performance capabilities, introduces many uncertainties in the engineering design community on how emerging microelectronics will perform in space. The engineering design community is forever behind on obtaining and developing new tools and guidelines to mitigate the harmful effects of the space environment. Adding to this complexity is the push to use Commercial-off-the-Shelf (COTS) and shrinking microelectronics behind less shielding and the potential usage of unproven technologies such as large solar sail structures and nuclear electric propulsion. In order to drive down these uncertainties, various programs are working together to avoid duplication, save what resources are available in this technical area and possess a focused agenda to insert these new developments into future mission designs. This paper will describe the relationship between the Living With a Star (LWS): Space Environment Testbeds (SET) Project and NASA's Space Environments and Effects (SEE) Program and their technology development activities funded as a result from the recent SEE Program's NASA Research Announcement.
\end{abstract}

\section{SEE Overview}

The Space Environments and Effects (SEE) Program was formed by the National Aeronautics and Space Administration (NASA) in 1993 to support the growing need for the development and maintenance of a preeminent program in space environments and effects technology. This initiative is intended to provide a coordinated national focus for innovative technology development to support design,

*Member AIAA

This paper is declared a work of the U.S. Government and is not subject to copyright protection in the United States. development, and operation of spacecraft systems that will accommodate or mitigate effects due to the presence of the space environment. The program is unique in that it was initiated as a customer-driven and productoriented endeavor. Considerable effort was made to ensure that the potential industry, academia, and government agency users of the products were consulted and made an integral part of the program. Their assessment and prioritizing of future research needs for space environment definition and techniques for calculating the effects of the space environment on spacecraft systems forms the basis for the SEE Program's activities. This direct involvement of potential customers also ensures that the SEE Program sponsored research products are made available in a timely manner to those most concerned with the information, i.e. spacecraft designers and operators.

\section{SEE Program Objectives and Goals}

The objectives of the SEE Program are to collect, develop, and disseminate the SEE-related technologies required to design, manufacture, and operate more reliable, cost-effective spacecraft for the Government and commercial sectors. In order to satisfy these objectives, the SEE Program has developed the following goals:

- Advocate technology development, flight experiments, and databases by creating and maintaining:

- Engineering environments definitions

- Up-to-date engineering focused models

- Environmental and materials databases

- Engineering design guidelines

- Flight/ground simulation/technical assessments

- Integrated assessment tools

- Simplified access to modeling and assessment tools 
- Maintain cutting edge expertise in SEErelated technologies by:

- Coordination with other agencies, industry, and academia

- The incorporation of technical experts and specialists

- Sustained awareness of state-of-the-art SEE technologies

- Heighten the awareness of SEE significance and program capabilities through:

- Internet access

- Bulletin

- Displays

- Workshops

- Publications $1,2,3,4,5,6$

\section{SEE Program Structure}

While the SEE Program was established with NASA sponsorship, it was recognized from the start that the program's success would depend on its ability to interact with research activities of other agencies, industry, and academia. This interaction has now become one of the principal strengths of the SEE Program. The SEE Program focuses on bridging the gap between the science community and the engineering community by improving environment definitions and tools for spacecraft design and operations planning. ${ }^{\text {? }}$
The Space Environments and Effects Program is organized (Figure 1) to facilitate the implementation of the Program's mission, which is directed toward research, development, verification, and transferring SEE-related technologies to the aerospace customers. ${ }^{8}$ While the program functions under the direction and oversight of the NASA Office of Aerospace \& Technology Enterprise; Enabling Concepts and Technology Program at NASA Headquarters, the key to its ability to accomplish its mission is the Technical Working Groups (TWG's). These groups encompass the disciplines of electromagnetic effects \& spacecraft charging, materials and processes, meteoroid and orbital debris, ionizing radiation, neutral contamination, and ionosphere and thermosphere (ad hoc). Their activities and responsibilities include: (1) ensuring communication with working group members, (2) coordinating development, evaluation, and maintenance of working group technical content and research areas, (3) coordinating research and development of new technologies and design issues that impact their respective space environmental areas, (4) coordinating development and use of engineering tools, models, and databases, and (5) coordinating development of spacecraft design and test techniques and methodologies for accommodating or mitigating space environment effects.

\section{SEE Program Organization}

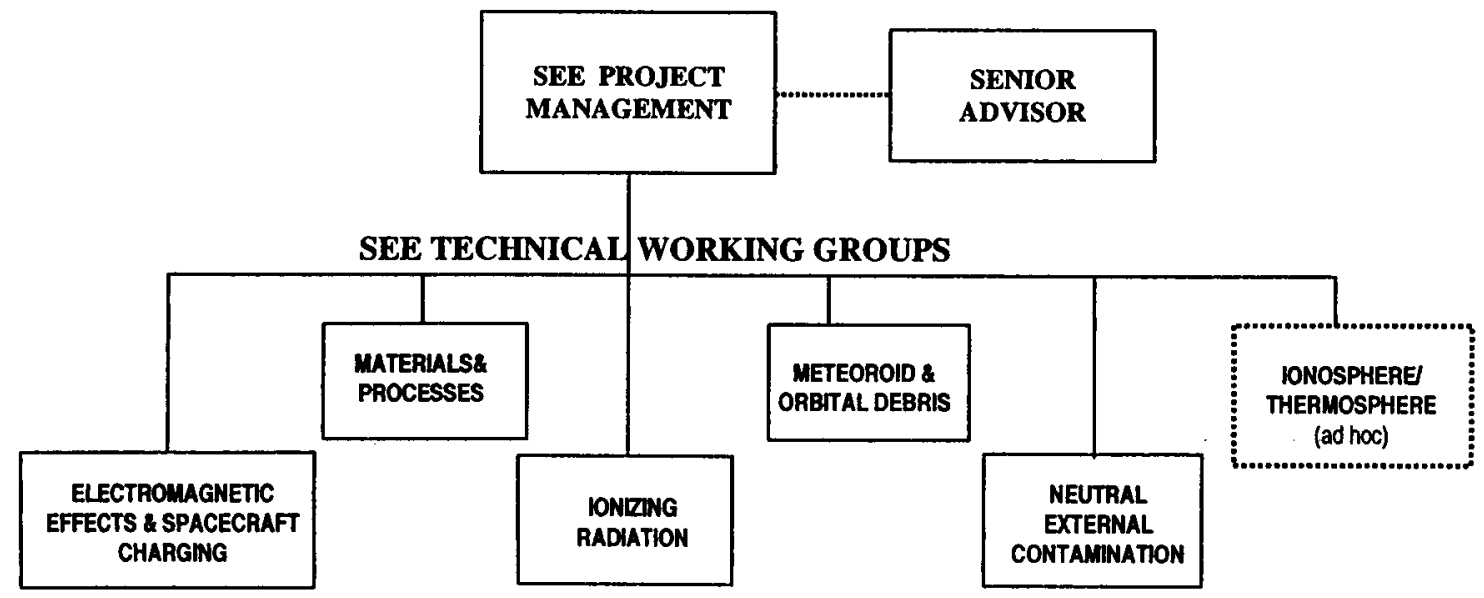

Figure 1. Space Environments and Effects Program Organization 
The membership in the SEE Technical Working Groups is drawn from NASA, Department of Defense (DoD), National Oceanic and Atmospheric Administration (NOAA), industry, and academia.

The six TWG's, as shown in Figure 1, are the Electromagnetic Effects \& Spacecraft Charging, Ionizing Radiation, Materials \& Processes, Meteoroid \& Orbital Debris, Neutral External Contamination, Ionosphere and Thermosphere (ad hoc) Working Groups. Members of these groups direct and, in some cases, conduct studies and tests that meet a recognized need of the customer. The data from these studies are incorporated into databases, design guidelines, and/or models and provided to the customers. The customers, through the Steering Committee, define systems requirements and needs to the Technical Working Groups and the program.

\section{LWS SET Project Description}

The LWS Program has the goal to develop a better scientific understanding to address the aspects of the connected Sun-Earth system that affect life and society. The Goddard Space Flight Center (GSFC) implements the LWS Program for the NASA Office of Space Science (OSS). A principal goal of the program is to bridge the gap between science, engineering, and user application communities as illustrated in Figure 2 .
The LWS Program is comprised of three elements: Science Missions to make scientific observations of the Sun, Heliosphere, and Geospace regions; Targeted Research and Technology (TR\&T); and the Space Environment Testbed (SET) Project. The Science Missions will collect the data required to achieve better understanding of the environment. The TR\&T program will develop new instrument techniques, models, and concepts for investigating solar and geospace disturbances; improve scientific knowledge of space environment conditions and variations over the solar cycle; improve understanding of the effects of solar variability on long-term climate change; and improve the environment specification models and predictive capability. The LWS SET Program will achieve understanding of the effects of solar varying environments on spacecraft, i.e., the induced environment. As new technologies are developed, space environment effects must be addressed before they can be used on space systems. The mechanisms of the interactions must be identified and modeled and ground test protocols to qualify technologies for space must be developed. The models and ground test protocols require flight validation to decrease safety margins and assure reliability.

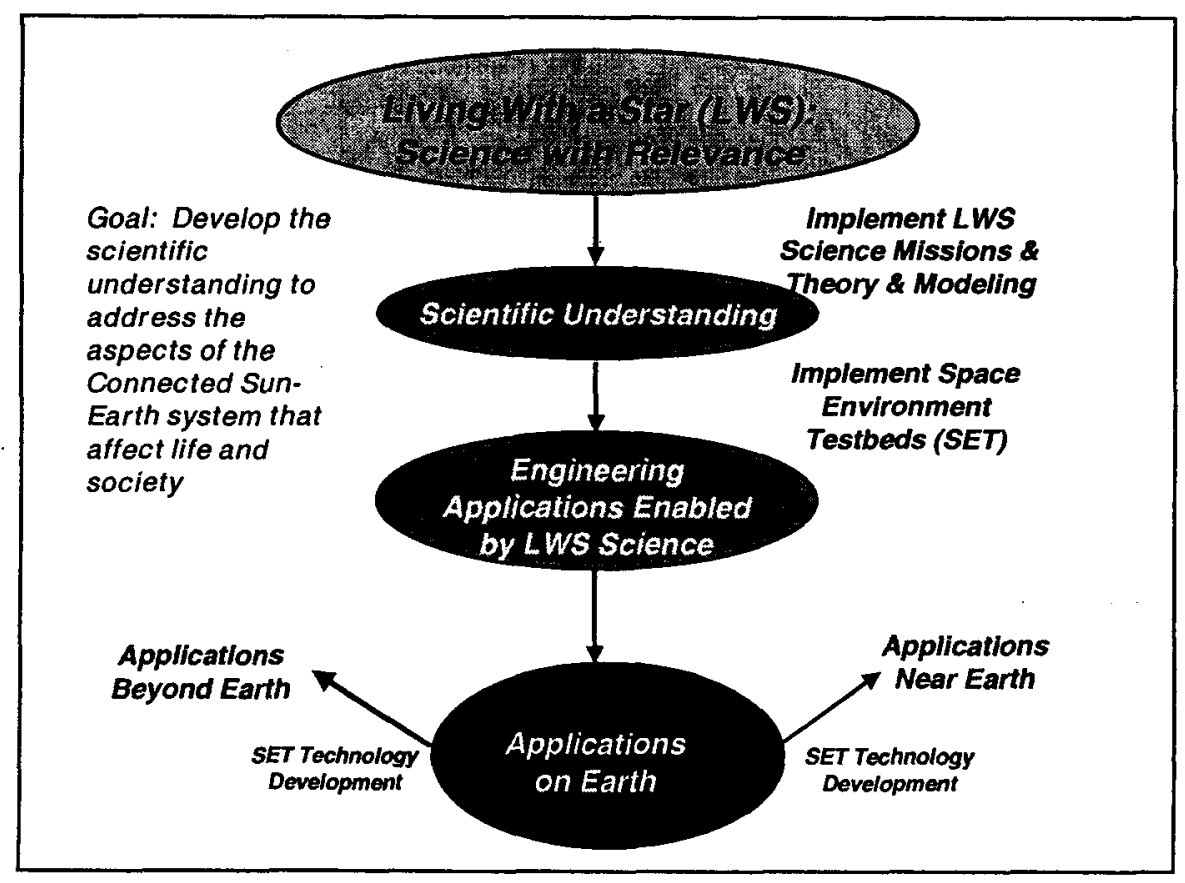

Figure 2. Living With a Star Concept 
The goal of SET is to improve the capability to accommodate or mitigate the effects of solar variability on spacecraft and instrument design and operations. To accomplish that goal, the SET has three objectives. The first is to improve the scientific understanding in four categories of space environment effects and mechanisms:

- Detector/sensor degradation and failure mechanisms;

- Methods to control/eliminate plasma effects;

- Methodologies to improve microelectronics performance; and

- Characterization of the space environment in the presence of a spacecraft.

Only those technologies that are subject to the variability of the space environment, and that need to be directly validated in that environment are of interest to the Program.

The second objective is to reduce design margins thereby increasing the use of space environment "tolerant" technologies, increasing the fraction of resources for payload, reducing launch vehicle requirements, and enabling routine operations above Low Earth Orbit (LEO) (above $2000 \mathrm{~km}$ ) for LEO cost. The third objective is to improve operational guidelines to reduce unnecessary shutdowns, lower the risk of anomalies, and reduce the number of failures.

The results of the first LWS SET NRA are presented in this paper. The focus of the NRA was:

Improve, develop, and validate engineering environment prediction and specification models, tools, and databases for reliable spacecraft design and operations in radiation, spacecraft charging, meteoroid, orbital debris, and thermosphere/ionosphere environments.

\section{SEE \& SET Program Relationship}

Since the SEE Program had been established since 1993, was firmly planted into the community and already possessed a close working relationship with the SET Project personnel, it made for a perfect fit for the SET Project to leverage the SEE Program in the technical areas that were related to the SEE Program In order to ensure there was no duplication, the SET Project incorporated SEE Program personnel into their organization and asked for the SEE Program to manage and distribute the products to the applications community from the technology development activities in the area previously described (improve, develop, and validate engineering environment prediction and specification models, tools, and databases).

All of the SEE Program activities (some recently completed in the past year) and SET Project activities (managed by the SEE Program) are described below.

\section{Technology Development Activities}

Since its inception in 1993, the SEE Program has released three separate NASA Research Announcements (NRA's) for technology development. To date, fifty contracts have been awarded, using peer review, with the technical disciplines represented including all those encompassed by the SEE Technical Working Groups. Organizations receiving the contracts included industry, academia, and government laboratories. The products of these three-year contract efforts from the 1994 and 1997 NRA's are now being realized and as the reports are received and processed, they are being distributed to the aerospace community through the SEE Program. ${ }^{10}$ Additional information concerning the SEE Program's available products can be obtained by contacting the author by email at billy.kauffman@msfc.nasa.gov.

The SET Project released its first solicitation within the third SEE Program NRA (July 2001) that produced eight one-year technology development activities. This announcement was a joint technology solicitation for NASA's SEE Program and LWS/SET Project with each program identifying individual focus areas.

\section{SEE Program}

\section{Meteoroids and Orbital Debris}

Title: Origin of the Projectile Residues in the Largest LDEF Craters

Organization: Johnson Space Center

Principal Investigator: Friedrich Horz

Objective: This effort will measure the composition of impactor residue in 
approximately 100 of the largest craters recovered from the LDEF Experiment S0001. The knowledge of meteoroids and man-made debris that can be derived from this effort, with the unique LDEF materials, is critically needed to establish impact risks to future spacecraft. The study leverages on the large investments already made in the LDEF program and complements the studies of larger size particles being made with ground-based sensors.

Title: Determination of Meteoroid Masses, Densities, and Ballistic Coefficients Using Decelerations Obtained from Multi-Frequency Radar and Optical Data

Organization: Marshall Space Flight Center Principal Investigator: Robert Suggs Objective: This effort will estimate the densities of ablating meteoroids entering the Earth's atmosphere from measurements of their decelerations. The result will be incorporated into a database that will have significant value to spacecraft designers in the development of spacecraft meteoroid protection systems.

Title: Meteoroid Engineering Environment for the Inner Solar System (MEEDISS)

Organization: University of Western Ontario; Marshall Space Flight Center

Principal Investigator: Jim Jones

Objective: Development of a universal model applicable to Earth-orbiting and interplanetary spacecraft for "sporadic" meteoroids. Model is physics-based so Earth observations are only used for validations. Model allows for extrapolation to other locations that is not possible with existing empirical models.

\section{Ionizing Radiation}

Title: Non-Ionizing Energy Loss (NIEL) Tool for Space Applications (co-funded with LWS SET Program)

Organization: Goddard Space Flight Center Principal Investigator: Michael Xapsos Objective: Provide a computer program for calculating NIEL in elemental and compound semiconductors for electrons, protons and heavy ions having energies relevant to space applications; and calculate NIEL spectra equivalent to linear energy transfer (LET) spectra for space environment of concern, e.g. galactic cosmic rays and solar particle events.

Title: Improved Solar Particle Risk Assessment Tool
Organization: Goddard Space Flight Center Principal Investigator: Michael Xapsos Objective: Develop an improved solar particle risk assessment computer program for spacecraft design. The corresponding environmental results will be coupled to a state-of-the-art transport code to allow analysis of the effect of spacecraft shielding on incident solar particle radiation.

Title: Design Guidelines for Ionizing Radiation Organization: SAIC; Goddard \& Marshall Space Flight Centers

Principal Investigator: Hugh Anderson; Jim Howard; Donna Hardage

Objective: Develop and publish ionizing radiation guidelines that will focus on the Program Manager, Design Engineer, and Space Radiation Engineer (0-5 yrs exp).

Spacecraft Charging (Plasma)

Title: Electric Propulsion Interactions Code (EPIC): Integrated Guidelines and Tools for the Assessment of Electric Propulsion Impact on Spacecraft

Organization: Science Applications International Corporation (SAIC)

Principal Investigator: Myron Mandell Objective: To enhance and combine existing plasma simulations codes into an integrated package, EPIC, to provide system designers with a tool for assessing electric propulsion spacecraft interactions.

Title: Measurement of Charge Storage Decay Time and Resistivity of Spacecraft Insulators Organization: Utah State University Principal Investigator: J.R. Dennison Objective: Investigate the methods for measurement of charge storage and the magnitude of the effect in typical space applications through a combination of straightforward measurements and reference to existing theory on conduction in insulators. Specifically: (i) perfect methods and test protocols to study and quantify the effect, (ii) perform ground-based measurements of the effect in a limited number of controlled studies on near-term, ground-based measurement of the effect in a limited number of controlled studies on near-term missions and (iii) extend existing preliminary work to develop improved techniques so that a wider array of materials and their parameters can be efficiently tested in multiple-sample testing procedures. 
Title: Electronic Properties of Materials Applicable to Spacecraft Charging Organization: Utah State University Principal Investigator: J.R. Dennison Objective: Determine the electrical properties for a wide array of spacecraft materials and integrate this new information into relevant NASA databases and models. Specifically, measure secondary and backscattered electron total yields from electron-induced, ion-induced and photon-induced energies.

Title: NASCAP-2K

Organization: SAIC

Principal Investigator: Myron Mandell

Objective: A five year, collaborative effort with U.S. Air Force to develop a comprehensive revision to NASA's spacecraft charging analysis code. The code will possess new algorithms for LEO, GEO, polar orbits, deep-dielectric charging, radiation belts and interplanetary space. It will also possess a new interactive Object Oriented Toolkit.

Title: Low-Earth Orbit (LEO) Spacecraft Charging Guidelines

Organization: Glenn Research Center Principal Investigator: Dale Ferguson Objective: Develop and publish a LEO spacecraft charging guideline and incorporate new test data as necessary.

Title: L2 Charged Particle Environment Model and Extreme Event Case Studies

Organization: Jacobs Sverdrup

Principal Investigator: Joe Minow; Bill Blackwell

Objective: Develop a semi-empirical engineering model of electrons and ions (from a few 100 's eV to approximately $1 \mathrm{MeV}$ ) that include the thermal plasma distributions as well as bulk flow effects in the distant magnetotail.

Title: Variability in LEO Plasma Environment Organization: Jacobs Sverdrup Principal Investigator: Joe Minow Objective: Develop a model using a plasma variability database to specify statistical variations of plasma parameters ( $\mathrm{Ne}, \mathrm{Te}, \mathrm{Ni}, \mathrm{Ti}$ ) in LEO environments (approximately $200 \mathrm{~km}$ to $2000 \mathrm{~km})$.

\section{Materials and Processes}

Title: Improved thermal Control Coating Development

Organization: Air Force Research Lab (AFRL)
Principal Investigator: Michael Donley Objective: Develop new thermal control coatings that have improved initial performance and significantly better end-of-life (EOL) performance over the desired service lifetime by improving the diffuse reflectance efficiency, decreasing UV absorbance and improving the physical/mechanical properties of the coating.

Title: In-situ Material Properties Sensor Organization: AZ Technology, Inc. Principal Investigator: Donald Wilkes Objective: Develop a small, compact optical properties measurement sensor system that can be configured for a wide range of uses for space experiments and ground-based tests.

Title: State-of-the-Art Materials Knowledgebase

Organization: CAT Flight Services; Computer Sciences Corporation; Marshall Space Flight Center

Principal Investigator: Larry Bradford; Sopo Yung; Jody Minor

Objective: Development of a comprehensive, single location for materials knowledgebase of thin film materials, ultra-lightweight structures and advanced adhesives.

\section{Neutral External Contamination}

Title: Material Additions to the Satellite Contamination and Materials Outgassing Knowledgebase

Organization: Bob Wood Aerospace

Consulting Services, Inc.

Principal Investigator: Bob Wood

Objective: Provide additional quartz-crystal microbalance (QCM) data to the Satellite Contamination and Materials Outgassing Knowledgebase. There is now the opportunity to essentially double the amount of E1559 information. This would bring the number of materials characterized to approximately 500 .

\section{Ionosphere and Thermosphere}

Title: Geospace Environment Implications for Spacecraft

Organization: Hanscom Air Force Research Lab; Space Environment Technologies

Principal Investigator: Frank Marcos \& Dwight Decker; Kent Tobiska Objective: Develop density and solar proxy databases from orbital drag data and improve solar EUV inputs to replace F10.7 in MET to 
improve MET density versus altitude and latitude. Develop a high-latitude scintillation and forecast tool.

\section{SET Project}

\section{Microelectronics}

Title: Analysis of CRRES PHA Data for LowLinear Energy Transfer (LET) Events

Organization: Clemson University Principal Investigator: Peter McNulty Objective: Single event upsets caused by trapped, galactic, and solar ions cause failures in microelectronics. This effort will analyze pulse height data from an experiment on CRRES, focusing on the measured low energy single events.

Title: Mining Enhanced Low Dose Rate Sensitivity (ELDRS) Data from the MPTB Space Experiment Organization: NAVSEA Crane Principal Investigator: Thomas Turflinger Objective: ELDRS effects in linear bipolar integrated circuits means that devices degrade more at low space dose rates than at accelerated ground test rates. Since virtually all space systems use bipolar linear integrated circuits, understanding the behavior of these parts in space is extremely useful to system designers.

\section{Detectors}

Title: Displacement Damage Effects in Solar Cells

Organization: Naval Research Laboratory Principal Investigator: Robert Walters Objective: Solar cell degradation due to protons and electrons reduces mission lifetime unless margins are added. In addition, the current codes used to calculate solar cell degradation are not adequate for state-of-the-art solar cells. This effort includes modifying the Solar Array Verification and Analysis Tool (SAVANT) to predict on-orbit degradation of multi-junction solar cells.

Title: Modeling Charge Collection in Detector Arrays Organization: PR\&T

Principal Investigator: James Pickel Objective: Noise transients on optical sensors due to charged particles cannot be accurately predicted with current models because they do not account for the energy deposition by low energy particles and charge collection by driftassisted diffusion. This effort provides for the development a more accurate model for on-orbit transient effects in detectors and will include drift assisted charge diffusion.

\section{Spacecraft Charging}

Title: Characterization of Magnetospheric Spacecraft Charging Environments Using the LANL Magnetospheric Plasma Analyzer Data Set

Organization: Science Applications International Corporation

Principal Investigator: Victoria Davis Objective: Spacecraft charging due to diurnal variations in the plasma environment produces spacecraft anomalies and failures. The current models of the plasma environment are not correlated to spacecraft charging events. This effort will identify those parameters that are important to spacecraft charging and allow for accurate predictive charging capabilities.

Degradation \& Shielding Properties of Materials

Title: Study of Total Ionizing Dose of High-Z Material Spot Shields on Field Programmable Gate Arrays (FPGA) Using Flight Data from Experiment Board on the Microelectronics and Photonics Testbed

Organization: The Aerospace Corporation Principal Investigator: Susan Crain Objective; It has been observed that the spot shielded FPGAs on MPTB show significantly more total ionizing dose degradation caused by protons and electrons than those with no spot shielding. This effort entails analyzing this behavior using concurrent radiation environment measurements, radiation environment models, and transport codes.

\section{Conclusions}

The NASA Space Environments and Effects (SEE) Program, in cooperation with the LWS SET Program since 2001, has made significant progress since 1993. As this paper illustrates, the SEE Program reflects an interagency and broad industry scope. The program also plays an important role as an advocate for space environments and effects related flight experiments. The program's success, however, depends upon the feedback from aerospace industry and government programs on their 
anticipated needs and the value of the program's products in their spacecraft systems development activities.

Those having interest in the SEE and LWS SET Program activities, whether from opportunities for participation, information on products, or to contribute inputs on future spacecraft needs relative to space environment definition or effects of the space environment on spacecraft systems, are invited to visit the websites homepages at $h t t p: / / s e e . m s f c . n a s a . g o v$ and http://ws-set.gsfc.nasa.gov/ respectively.

\section{Acknowledgements}

The authors wish to acknowledge the contributions of Steve Pearson, Senior Advisor, at NASA's Marshall Space Flight Center. 


\section{References}

1. Belk, C. A., J. H. Robinson, M. B. Alexander, W.J. Cooke, and S. D. Pavelitz (1997): "Meteoroids and Orbital Debris: Effects on Spacecraft." NASA Reference Publication 1408.

2. Herr, J. L. and M. B. McCollum (1994): "Spacecraft Environments Interactions: Protecting Against the Effects of Spacecraft Charging." NASA Reference Publication 1354

3. Leach, R. D. and M. B. Alexander (1995): "Failures and Anomalies Attributed to Spacecraft Charging." NASA Reference Publication 1375.

4. Vaughan, W. W., K. O. Niehuss, and M. B. Alexander (1996): "Spacecraft Environments Interactions: Solar Activity Effects on Spacecraft" NASA Reference Publication 1396.

5. James, B. F, O. A. Norton, Jr., and M. B. Alexander (1994): "The Natural Space Environment: Effects on Spacecraft." NASA Reference Publication 1350.

6. Bedingfield, K. L., R. D. Leach, and M. B. Alexander(Editor) (1996): "Spacecraft System Failures and Anomalies Attributed to the Natural Space Environment." NASA Reference Publication 1390.

7. Pearson, S.D., B. Kauffman, S. Clifton and C. Upton, "Developing Tomorrow's Space Technology Today; NASA's Space Environments and Effects (SEE) Program," AIAA 964374, AIAA Space Programs and Technologies Conference, Huntsville, AL, September 24 -26, 1996.

8. Hayduk, R.J. and S.D. Pearson, "NASA's Space Environments and Effects Program Technology Development and Risk Mitigation for Spacecraft Design," IAF-97-I.3.03, 48 ${ }^{\text {th }}$ International Astronautical Congress, Turin, Italy, October 6 - 10, 1997.
9. Pearson, S.D., K.S. Clifton and W.V. Vaughan, "NASA's Space

Environments and Effects Program and Related Space-borne Experiments," AIAA 98-0294, 36 ${ }^{\text {th }}$ Aerospace Sciences Meeting and Exhibit, Reno, NV, January $12-15,1998$.

10. Pearson, S.D., K.S. Clifton and W.V. Vaughan, "NASA's Space Environments and Effects Program and Related Space-borne Experiments," AIAA 98-0294, $36^{\text {th }}$ Aerospace Sciences Meeting and Exhibit, Reno, NV, January $12-15,1998$. 